\title{
Mice generated after round spermatid injection into haploid two-cell blastomeres
}

Cell Research (2011) 21:854-858. doi:10.1038/cr.2011.45; published online 22 March 2011

\section{Dear Editor,}

It is well known that mature oocytes can reprogram somatic nuclei in the process of nuclear transfer (NT) [1] and remodel male gametes in normal fertilization. An intriguing question is whether blastomeres from cleavagestage embryos retain reprogramming activities. Recently, Egli et al. [2] reported that one blastomere of the twocell-stage embryo can successfully reprogram a donor nucleus, resulting in the formation of an embryonic stem (ES) cell line or a chimeric mouse. Therefore, it is interesting to investigate whether two-cell blastomeres can remodel male gametes as mature oocytes do. Using mature sperm and round spermatid as donors, we found that two-cell blastomeres can partially remodel mature sperm, but can completely remodel round spermatids to produce blastocysts carrying male chromosomes in all the cells, from which normal functional ES cells can be generated. Our results demonstrate that remodeling ability persists in the blastomeres of two-cell embryos.

To determine whether the two-cell blastomere can remodel male gametes to generate normal diploid embryos, we produced haploid two-cell blastomeres as recipients. Diploid parthenogenetic embryos can be achieved conventionally by activation of MII oocytes in the presence of cytochalasin $\mathrm{B}(\mathrm{CB})$, which prevents extrusion of the second polar body. To derive the haploid two-cell-stage embryos, CB was removed from the activation medium. In the control experiments, a total of 109 oocytes were activated for $6 \mathrm{~h}$ in CB-free activation medium. Most of the oocytes (104/109) extruded the second polar body and formed one pronucleus. Although all of them divided into the two-cell stage, only $14 \%(15 / 104)$ developed into blastocysts, consistent with a previous report [3]. The other five oocytes, which did not extrude the second polar body and formed two pronuclei, developed into blastocysts. Next, we analyzed the cell-cycle stage of haploid two-cell embryos by culturing them in medium containing 5'-bromodeoxyuridine (Brdu). We observed that two-cell embryos usually formed at $18 \mathrm{~h}$ and entered the $\mathrm{S}$ phase at $19 \mathrm{~h}$ after parthenogenetic activation.
To trace the fate of injected sperm, we isolated sperms from homozygous transgenic mice expressing the enhanced green fluorescent protein (GFP) from the chicken $\beta$-actin promoter (EGFP mice). Intracytoplasmic sperm injection (ICSI) into one blastomere of a two-cell embryo (Supplementary information, Figure S1A) was performed as described $[3,4]$. Of 86 injected haploid two-cell embryos, 17 developed into blastocysts. However, none of them were green fluorescent, indicating that sperms did not participate in blastocyst development. We then investigated the remodeling of sperm chromatin by staining injected embryos with the DNA dye Hoechst 33342. We found that sperm heads began to undergo decondensation at $16 \mathrm{~h}$ after injection when the blastomere entered mitosis (2 of 15 , or $13 \%$ ) (Supplementary information, Figure S1B). With most of the blastomeres entering into mitosis at $18 \mathrm{~h}$ after injection, we observed more expanded sperm heads ( 15 of 20 , or $75 \%$ ) in the cytoplasm of blastomeres (Supplementary information, Figure S1C). Shortly after mitotic entry, the embryos divided into the four-cell stage. Following staining, we observed that in 7 of 15 four-cell embryos, one blastomere contained two nuclei (Supplementary information, Figure S1D). These results indicate that sperm chromatin can be decondensed by two-cell embryos in mitosis when the nuclear envelope is broken down and undefined remodeling factors are released into the cytoplasm.

Since two-cell embryos divide into the four-cell stage very quickly after entering mitosis, exposure of sperm head to remodeling factors might be too short to support the preimplantation development of injected embryos. We thus blocked the injected blastomere in mitosis for 6 $\mathrm{h}$ using nocodazole as described [2], and found that $3 \%$ of injected embryos ( 2 of 69) developed into blastocysts, in which some cells expressed GFP (Supplementary information, Figure S2A-S2D), indicating that they carried the sperm chromatin. Alternatively, the amount of remodeling factors in one blastomere might not be sufficient to remodel sperm chromatin. To address this problem, we fused the two blastomeres following sperm head injection (Supplementary information, Figure S3A). We 
observed that sperm head was not decondensed until the fused embryo entered into mitosis and $25 \%$ of injected embryos (5 of 20) had one blastomere carrying two nuclei at the two-cell stage (Supplementary information, Figure S3B and S3C). From 105 manipulated embryos, we obtained three blastocysts expressing GFP partially (Supplementary information, Figure S3D). Taken together, these data strongly suggest that factors present in the nucleus of blastomeres can remodel the sperm chromatin. However, in these sets of experiments, sperm head, which was decondensed only when recipient blastomere entered into mitosis, transformed into a male nucleus in one of two descendants of the injected blastomere in the next cell cycle. Therefore, by injection of mature sperm into the blastomere of two-cell embryos, we could not generate a blastocyst in which all cells contain sperm chromosomes. For this reason, we could not exclude the possibility that the development of injected blastomere depended on the blastomeres that did not carry sperm genome.

Round spermatids contain a haploid set of chromosomes, which are not condensed and lack protamines. After injection into oocytes, they can support the development of oocyte into normal fertile offspring [3-5]. We then performed round spermatid injection (ROSI) into a two-cell blastomere according to the methods described by Kishigami et al. [3]. We isolated round spermatids from the testis of EGFP male mice and injected one spermatid into one blastomere of the haploid two-cellstage embryo (Supplementary information, Figure S4A). Interestingly, we observed two nuclei in some embryos (4 of 20 , or $20 \%$ ) $10 \mathrm{~h}$ after injection, suggesting that the nucleus of the round spermatid, in contrast to mature sperm, might have undergone remodeling (Supplementary information, Figure S4B-S4D) before blastomere enters mitosis. These observations imply that the male nucleus can fuse with the female nucleus before entry into mitosis, resulting in all descendants of the injected blastomere possessing the male genome. To optimize ROSI into blastomere, we injected a green-fluorescent round spermatid into one blastomere at different time points after activation and followed the developmental potential of these embryos to blastocysts. We found that the best blastocyst development rate was achieved when twocell-stage embryos in G1 phase (18 h post activation) were used as recipients ( 8 green-fluorescent blastocysts derived from 70 manipulated two-cell embryos, or 11\%) (Supplementary information, Figure S4E).

To exclude the possibility that the uninjected blastomere supported the development of the injected one, we performed two independent sets of experiments. First, we fused the two blastomeres following ROSI (Supplemen- tary information, Figure S5A). Remodeling of the round spermatid chromatin was judged to have occurred by the appearance of three nuclei in the fused embryos $10 \mathrm{~h}$ after injection (4 of 20, or $20 \%$, Supplementary information, Figure S5B), and of two nuclei in one blastomere of two-cell embryos $16 \mathrm{~h}$ after injection (8 of 20,40\%, Supplementary information, Figure S5D and S5E). We cultured 95 manipulated embryos in vitro and obtained 40 blastocysts, in which 9 displayed green fluorescence in all the cells and 18 expressed GFP partially (Supplementary information, Figure S5C and S5F). This set of experiments demonstrates that two-cell blastomeres sustain the remodeling ability, but the resultant whole greenfluorescent embryos are triploid, and thus not amenable to further functional test. Therefore, in the second set of experiments, we injected one green-fluorescent round spermatid into each of the two blastomeres (Figure 1A). From 256 successfully injected embryos, we generated 83 blastocysts, in which 45 were not green fluorescent and 30 displayed green fluorescence partially. The remaining eight blastocysts ( $3 \%$ of manipulated embryos) expressed GFP in all the cells (Figure 1B), further demonstrating that both blastomeres of the two-cell embryo could remodel spermatid nucleus to support development to the blastocyst stage.

In NT studies, pre-implantation development of the reconstructed embryos into blastocysts is an initial, but not definitive measure of functional nuclear reprogramming [6], since most of the reconstructed blastocysts cannot generate viable mice or ES cells [7]. To test whether the generated blastocysts were functional, we cultured the eight whole green-fluorescent blastocysts on feeder cells in the ES culture medium. Four ES cell lines (1.6\% of manipulated embryos) were derived and displayed intense green fluorescence in all the cells (Figure 1B). Immunostaining of these ES cells revealed the presence of markers characteristic of mouse ES cells (Figure 1C). Karyotyping analysis of these ES cells revealed that three of them had 40 mouse chromosomes (Figure 1D), and the other one had 60 chromosomes. (It confirmed our earlier observations that two pronuclei can be formed in a fraction of parthenogenetic embryos activated in CBfree medium.) To test the developmental potential of the ES cells with the normal karyotype, we injected them into diploid blastocysts. Healthy chimeric mice were derived and were successful in germ-line transmission (Figure 1E). Next, we asked whether the ES cells can contribute toward all the embryonic lineages, the most stringent test of pluripotency, by injection of ES cells into tetraploid blastocysts [8]. A total of 220 tetraploid blastocysts injected with ES cells (passage 5) from one cell line resulted in the birth of nine viable pups, which 
A

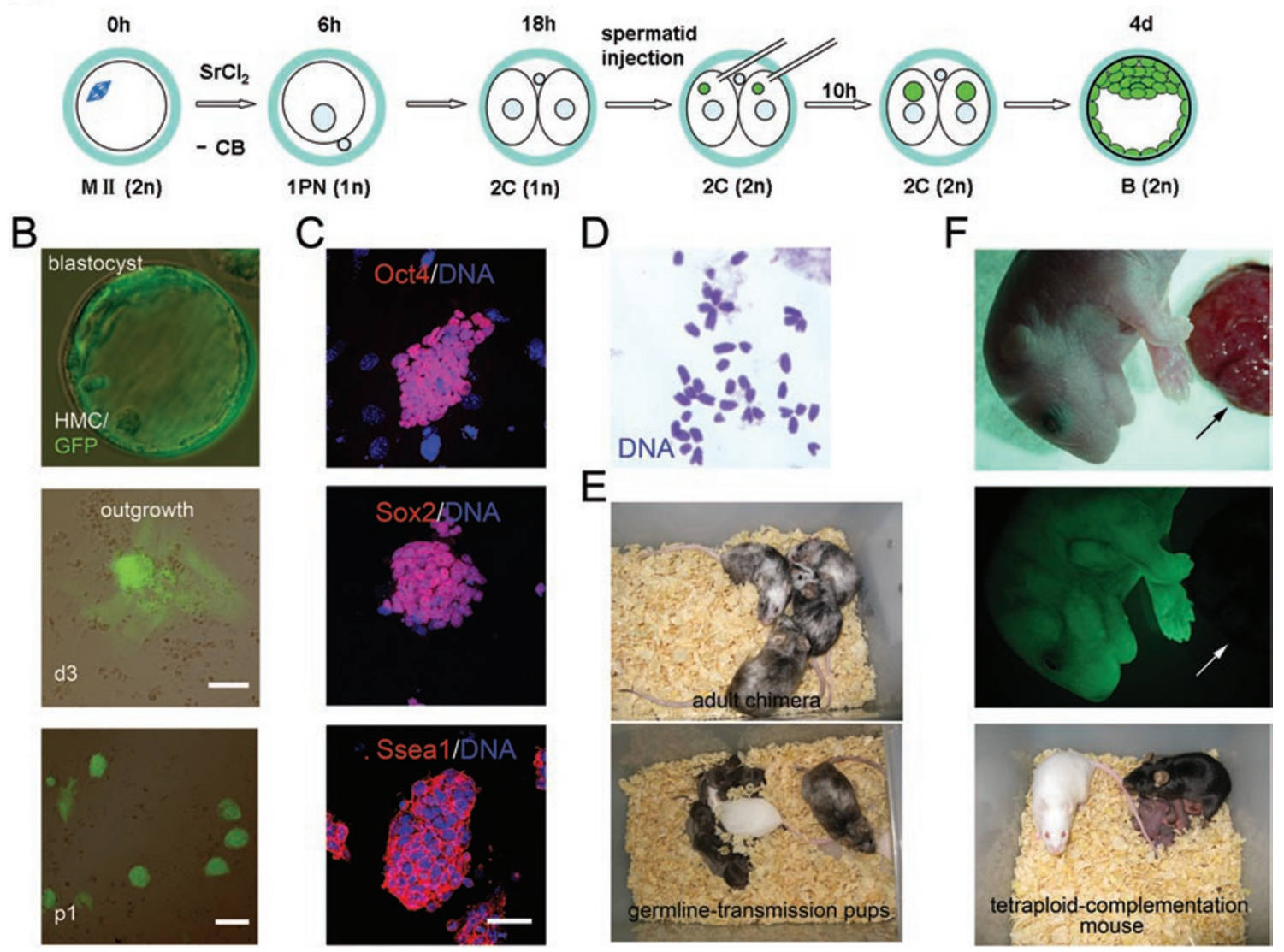

Figure 1 ES cells generated after ROSI into both blastomeres of haploid two-cell embryos. (A) Experimental design. MII oocytes, activated in CB-free medium for $6 \mathrm{~h}$, form one pronucleus and develop to two-cell stage in vitro. Green-fluorescent round spermatid is injected into both blastomeres. Injected embryos develop to blastocysts, in which all cells are green fluorescent. (B) ES cells derived from green-fluorescent blastocyst. All cells are green fluorescent in blastocyst (top), outgrowth (middle, scale bar, $50 \mu \mathrm{m}$ ) and ES cells (bottom, scale bar, $100 \mu \mathrm{m}$ ). (C) Expression of pluripotency markers in ES cells. Immunostaining with antibodies against Oct4 (top), Sox2 (middle) and Ssea1 (bottom). Counterstain was performed with blue nuclear dye Hoechst 33342. Scale bar, $50 \mu \mathrm{m}$. (D) Normal karyotype of ES cells. (E) Healthy chimeric mice generated from ES cells (top) exhibited germline-transmission (bottom). (F) Mouse produced by injection of ES cells into tetraploid blastocysts. Top, mouse and placenta, bright-field image. Middle, same mouse and placenta, fluorescent image. Mouse is green fluorescent. Arrow indicates the placenta, which is host-derived and thus not green fluorescent. Bottom, ES mouse (black) at 2 months with new-born pups.

are referred to as chimeric mice to reflect the possibility of tetraploid cell content in the developing fetus or newborn mice $[9,10]$. Of the live-born chimeric mice, four died within $1 \mathrm{~h}$. The other five mice rapidly gained active movement after birth, but four of them were eaten by the foster mother in one day and one pup survived to adulthood (Figure 1F). These results suggest that ES cells derived from blastocysts, which were generated after injection of round spermatids into both blastomeres of haploid two-cell embryos, exhibit the same pluripotency as those of fertilization-derived ES cell lines.
Finally, to test whether reconstituted embryos generated after ROSI into both blastomeres of haploid two-cell embryos could develop to term in vivo, we transferred a total of 373 injected two-cell embryos into oviducts and a total of 139 GFP-positive blastocysts into the uterus of pseudopregnant recipient females. However, none of them developed to term. These data suggest that although functional ES cells can be generated after ROSI into haploid two-cell blastomeres, normal in vivo developmental ability is not established in the embryos reconstructed by our strategy. The formation of a male pronucleus is essential for the embryonic development. When male 
pronucleus, which formed in mature oocytes, was transferred into parthenogenetic haploid embryo at the late two-cell stage, the constructed diploid embryos could develop to term [11]. When male gametes were injected into oocytes at $100 \mathrm{~min}$ after activation, offspring production for both ICSI and ROSI decreased significantly [3]. Here, we found that ROSI into haploid two-cell embryos fails to develop to term. Taken together, normal in vivo development appears to require the formation of a male pronucleus at the right time.

The reprogramming ability of blastomeres of cleavage-stage embryos has been tested [12-15], but these blastomeres generally failed to display functional reprogramming, which can be judged by ES cell derivation or mice generation. Recently, Egli et al. demonstrated that one of the two-cell-stage blastomeres retains the reprogramming ability when the blastomeres are arrested in mitosis [2]. Here, we observed that both blastomeres of the haploid two-cell embryos could remodel the nuclei of round spermatids to produce ES cells, which are functionally the same as normal ES cells. Our results demonstrate with more certainty that blastomeres of two-cell embryos contain remodeling activities.

Compared to round spermatid, mature sperm is more difficult to be remodeled in the blastomere of the twocell embryo. Sperm heads could not be decondensed until blastomeres entered mitosis, while some of the round spermatids could form male nucleus before blastomere entry into mitosis. These observations suggest that both the cytoplasm and nucleus of two-cell blastomeres contain remodeling factors, in which cytoplasmic factors alone are sufficient to remodel round spermatid into male nucleus, but not sufficient to remodel the mature sperm, which also needs factors from blastomere nucleus. Therefore, it would be interesting to further discern between cytoplasmic factors and nuclear factors which of these are involved in these processes in the future.

\section{Acknowledgments}

We thank Dr Xiang Gao of Nanjing University for providing EGFP-transgenic mice. We thank Drs Yixian Zheng of Carneige Institutation and Haifan Lin of Yale University for their critical and useful comments on the manuscript. This work was supported by grants from the Chinese Academy of Sciences (KSCX2-YW-R-110, KSCX2-YW-R-229, XDA01010403), the Ministry of Science and Technology (2007CB947101, 2009CB941101), National Natural Science Foundation of China (30871430) and the Shanghai Municipal Commission for Science and Technology (07DZ22919, 08DJ1400502, 09PJ1410900).

Hui Yang ${ }^{1, *}$, Linyu $\mathrm{Shi}^{1{ }^{1,},}$, Charlie Degui Chen ${ }^{2,3}$, Jinsong $\mathrm{Li}^{1}$
${ }^{1}$ Laboratory of Molecular Cell Biology, Institute of Biochemistry and Cell Biology, Shanghai Institutes for Biological Sciences, Chinese Academy of Sciences, 320 Yueyang Road, Shanghai 200031, China: '25tate Key Laboratory of Molecular Biology, Institute of Biochemistry and Cell Biology, Shanghai Institutes for Biological Sciences, Chinese Academy of Sciences, $320 \mathrm{Yu}$ eyang Road, Shanghai 200031, China; ${ }^{3}$ Shanghai Key Laboratory of Molecular Andrology, Institute of Biochemistry and Cell Biology, Shanghai Institutes for Biological Sciences, Chinese Academy of Sciences, 320 Yueyang Road, Shanghai 200031, China

*These two authors contributed equally to this work.

Correspondence: Jinsong Li

Tel: +86-21-54921422; Fax: +86-21-54921426

E-mail: jsli@sibs.ac.cn

\section{References}

1 Meissner A, Jaenisch R. Mammalian nuclear transfer. Dev Dyn 2006; 235:2460-2469.

2 Egli D, Sandler VM, Shinohara ML, Cantor H, Eggan K. Reprogramming after chromosome transfer into mouse blastomeres. Curr Biol 2009; 19:1403-1409.

3 Kishigami S, Wakayama S, Nguyen VT, Wakayama T. Similar time restriction for intracytoplasmic sperm injection and round spermatid injection into activated oocytes for efficient offspring production. Biol Reprod 2004; 70:1863-1869.

4 Kimura Y, Yanagimachi R. Mouse oocytes injected with testicular spermatozoa or round spermatids can develop into normal offspring. Development 1995; 121:2397-2405.

5 Ogura A, Matsuda J, Yanagimachi R. Birth of normal young after electrofusion of mouse oocytes with round spermatids. Proc Natl Acad Sci USA 1994; 91:7460-7462.

6 Hochedlinger K, Jaenisch R. On the cloning of animals from terminally differentiated cells. Nat Genet 2007; 39:136-137; author reply 137-138.

7 Hochedlinger K, Jaenisch R. Nuclear reprogramming and pluripotency. Nature 2006; 441:1061-1067.

8 Jaenisch R, Young R. Stem cells, the molecular circuitry of pluripotency and nuclear reprogramming. Cell 2008; 132:567582.

9 Eakin GS, Hadjantonakis AK, Papaioannou VE, Behringer RR. Developmental potential and behavior of tetraploid cells in the mouse embryo. Dev Biol 2005; 288:150-159.

10 Li J, Ishii T, Wen D, Mombaerts P. Non-equivalence of cloned and clonal mice. Cur Biol 2005; 15:R756-R757.

11 Barra J, Renard JP. Diploid mouse embryos constructed at the late 2-cell stage from haploid parthenotes and androgenotes can develop to term. Development 1988; 102:773-779.

12 Eckardt S, Leu NA, Kurosaka S, McLaughlin KJ. Differential reprogramming of somatic cell nuclei after transfer into mouse cleavage stage blastomeres. Reproduction 2005; 129:547-556.

13 Roh S, Guo J, Malakooti N, Morrison JR, Trounson AO, Du ZT. Birth of rats following nuclear exchange at the 2-cell stage. Zygote 2003; 11:317-321.

14 Tsunoda Y, Yasui T, Shioda Y, Nakamura K, Uchida T, Sugie T. Full-term development of mouse blastomere nuclei trans- 
planted into enucleated two-cell embryos. J Exp Zool 1987; 242:147-151.
15 Solter D. Mammalian cloning: advances and limitations. Nat Rev Genet 2000; 1:199-207.

(Supplementary information is linked to the online version of the paper on the Cell Research website) 